\title{
Precise additive fabrication of wall structure on thin plate end with interlayer temperature monitoring
}

\author{
Takenao TSURUMAKI*, Shinji TSUKAMOTO**, Hiroyuki CHIBAHARA** and Hiroyuki SASAHARA* \\ ${ }^{*}$ Tokyo University of Agriculture and Technology \\ 2-24-16 Naka-cho, Koganei-shi, Tokyo 184-0012, Japan \\ E-mail: nextwelve0712@gmail.com \\ ** Mitsubishi Electric Corp., \\ 8-1-1 Tsukaguchi-Honmachi, Amagasaki-shi, Hyogo 661-8661, Japan
}

Received: 30 January 2019; Revised: 28 March 2019; Accepted: 31 March 2019

\begin{abstract}
Turbine blades need to be repaired to extend their product life, because they are used in highly heated centrifugal fields and often suffer damage such as cracks, chippings, and oxidation thinning. The objective of this study is to perform additive fabrication onto the thin end of a plate using wire and arc-based additive manufacturing and the conduct finish cutting to repair turbine blades made of Inconel 718. It was successfully demonstrated to fabricate a wall structure onto the thin end of a plate with a thickness of 1 to $5 \mathrm{~mm}$ with the aimed deposition width while maintaining a constant interlayer temperature. In addition, a finish cutting was conducted on the additionally fabricated wall structure to achieve the same thickness as the thin base plate. It was also shown that there was almost no uncut part on the additive wall surface.
\end{abstract}

Keywords: Wire and arc-based additive manufacturing, Cutting, Thin plate, Inconel 718, Interlayer temperature monitoring

\section{Introduction}

Inconel 718 has high temperature strength and corrosion resistance, and it is used for turbine blades such as those used in gas turbines (Thomas et al., 2006). However, turbine blades are used in highly heated centrifugal fields and often suffer damage such as cracks, chippings, and oxidation thinning (Carter, 2005, Rani et al., 2017). It is necessary to repair them to extend their product life (Liu et al., 2016). Normally, damaged parts are repaired manually with arc welding process, but the occurrence of welding defects due to manual variation in quality is a problem. There is an increasing expectation that additive manufacturing, in which only the required amount of metal material is added locally to repair an existing metal workpiece, may be one solution to this problem (Pallos, 2001, Bi and Gasser, 2011). According to GE power systems, the Weld Repair Advanced Process ${ }^{\mathrm{TM}}$ (WRAPTM), which is a new plasma arc welding method that prevents weld cracking, was developed and applied for the repair of turbine blades to facilitate their reuse (Pallos, 2001). According to $\mathrm{Bi}$ and Gasser, laser-aided additive manufacturing (LAAM), which is a laser metal deposition method used to repair nickel-based turbine blade knife-edges, was investigated, and they demonstrated that LAAM is a feasible way to recondition turbine blade knife-edges (Bi and Gasser, 2011). We focus on wire and arc-based additive manufacturing (WAAM). In contrast with WRAPTM and LAAM, it is expected that WAAM can be applied to repair mechanical parts at low cost and enhance performance by depositing the same metal material used in the mechanical parts using the common metal inert gas (MIG) or metal active gas (MAG) welding method.

Because thin metal structures such as turbine blades have a small heat capacity, the temperature in the vicinity of the fabrication zone can rise easily when the width of the additive fabricated part becomes large. This results in a decrease of shape accuracy. On the other hand, there arises a problem that the metal material does not melt, and the fabrication cannot be done when the heat input is small. Therefore, it is necessary to set the deposition conditions, such as the welding current, arc voltage, wire feed speed, torch feed speed, and interlayer temperature, appropriately. As 
shown in Fig. 1, the molten metal tends to drip, and additive fabrication cannot be performed because the plate thickness is so thin that it is difficult to ensure that the molten metal will stay at the thin end of the plate. Therefore, it is necessary to do the additive fabrication at deposition widths that are not excessively larger than the plate thickness. In addition, it is necessary to perform finish cutting on the additively fabricated part with the same precision exhibited before the repair because there are the steps between the layers of the fabrication which performed fabrication by WAAM. It is necessary to perform additive fabrication at deposition widths that take into consideration the steps between the layers of the fabrication so that uncut parts do not remain on the surface after cutting.

The objective of this research is to clarify the appropriate deposition conditions under which to perform additive fabrication and cutting on the thin end of a plate using WAAM in order to repair turbine blades using Inconel 718. The relationship between the deposition conditions and deposition widths by fabricating wall structures on substrates while maintaining a constant temperature in the interlayer using interlayer temperature monitoring were investigated. Based on the relationship between the deposition conditions and deposition widths, we attempted to perform additive fabrication onto the thin end of a plate with various plate thickness while maintaining a constant interlayer temperature to achieve the stable deposition. In addition, it was shown that the additively deposited wall structure on the thin end of the plate has some allowance to be removed on the finish cutting, so uncut region cannot be observed.

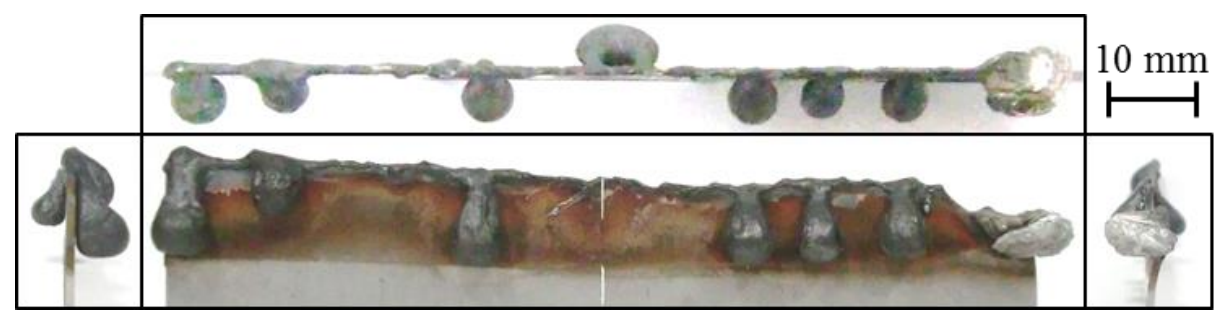

Fig. 1. Example of molten metal dripping in an additive fabrication onto the thin end of a plate

\section{Experimental procedure}

Figure 2 shows the WAAM machine used in this study (Abe and Sasahara, 2016). It is a 4-axis control machine (with axes X, Y, Z, and B) with a tilting axis (B axis), and the strokes of the X, Y, Z and B axes are $400 \mathrm{~mm}, 300 \mathrm{~mm}$, $150 \mathrm{~mm}$, and $\pm 90^{\circ}$, respectively. The maximum feed speed of each axis is $1000 \mathrm{~mm} / \mathrm{min}$. A MIG/MAG welding torch is attached to the Z-axis, and its moving path is numerically controlled. The fabrication will be built on the metal workpiece fixed on the base block.

Figure 3 shows the WAAM process mechanism. The welding wire acting as the material to be deposited is fed to the welding torch tip. An electric current is supplied through the contact tip, and an arc discharge is generated between the tip of the welding wire and the existing metal workpiece. As the molten metal is deposited due to the energy of the arc discharge, the welding torch moves on the XY plane to form a layer. This operation is repeated after the torch is raised along the $\mathrm{Z}$-axis to maintain a distance of about $10 \mathrm{~mm}$ between the welding torch tip and the deposition point after each layer is completed. While the arc is being generated, argon gas is released from the tip of the welding torch to shield the arc and weld metal from the atmosphere. The generation of welding defects and oxidation of the metal can be inhibited, and stable deposition can be achieved.

In WAAM wall structure fabrication, a subsequent layer is deposited onto the previous layer only after the temperature of the previous layer has been lowered to a certain temperature. The surface temperature of the previous layer is called as the interlayer (or interpass) temperature (Funderburk, 1998). One common method to know the interlayer temperature is to use two temperature-indicating crayons which have two kinds of crayons with different melting temperature. It can indicate the temperature the object between two melting temperature. However, with this method it is difficult to measure the exact interlayer temperature. On the other hand, there are two types of methods for directly measuring the interlayer temperature: a contact type measurement using a surface thermometer or a thermocouple; and a noncontact type measurement using an infrared thermography camera (Petro, 2011). However, with the contact-type method, only the surface temperature of the existing metal workpiece at a position adjacent to the welding bead can be measured. This method is not considered suitable for fabricating a wall structure in which the welding beads are deposited in the vertical direction. Thus, in this study, we monitored the interlayer temperature using an infrared thermography camera (SC620 and CPA-T650SC from FLIR Systems) to measure the surface temperature of 
the fabricated wall structure. The surface temperature at the center of the upper surface of the previous layer was measured as the interlayer temperature using the spot temperature measurement function. The emissivity of the fabricated wall structure of Inconel 718 was measured by applying a black body spray with an emissivity of 0.94 to the fabricated wall structure, and adjusting it so that the temperature of the applied part and that of the non-applied part were equal; the average emissivity value was determined to be 0.37 at about $40{ }^{\circ} \mathrm{C}$. As the emissivity is largely affected by the surface finish and oxidation, the fabricated object itself was used for the calibration. In addition, temperature dependence on the emissivity of Inconel 718 is small (del Campo et al., 2010), constant emissivity was employed in this study. The first layer was fabricated at room temperature without preheating the substrate. As an example of the interlayer monitoring, Figure 4 presents a thermal image of the fabricated wall structure at the first fabrication layer.

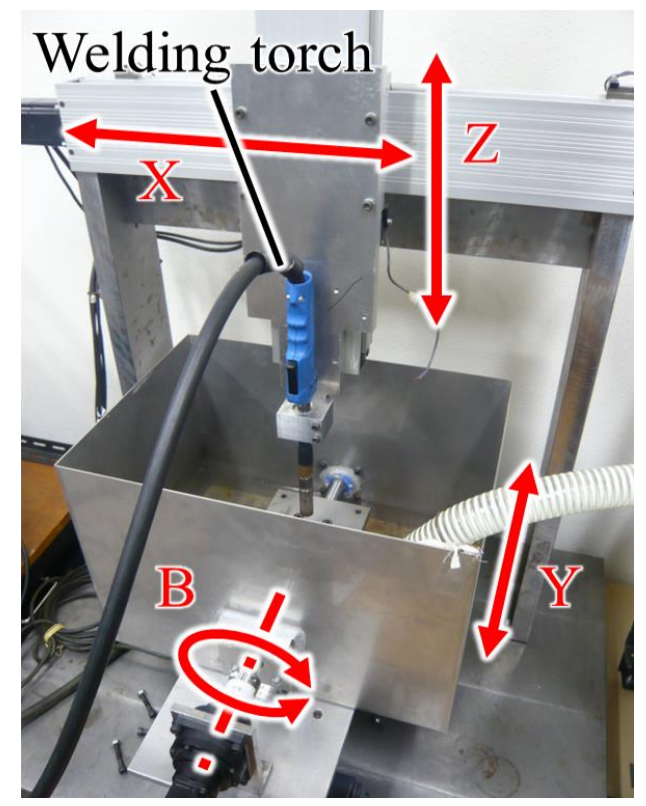

Fig. 2 Wire and arc-based additive manufacturing machine

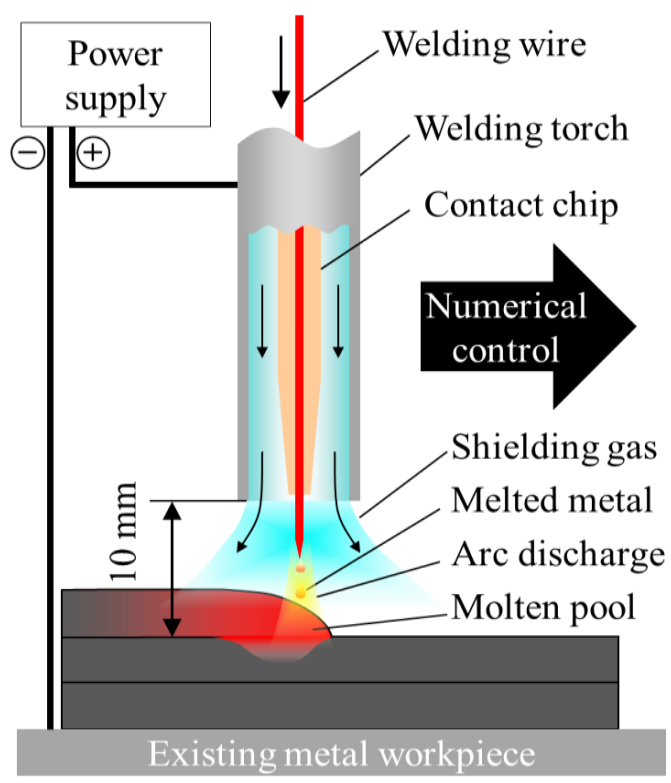

Fig. 3 Deposition mechanism

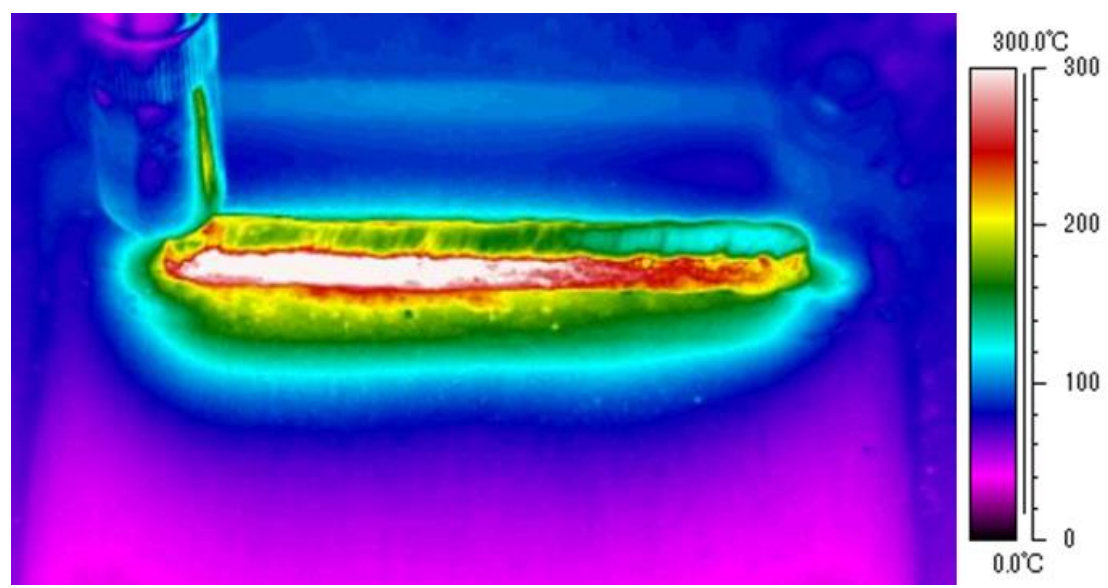

Fig. 4 Thermal image of additive fabrication

\section{Higher precision of deposition width by interlayer temperature monitoring}

In additive fabrication onto the thin end of a plate, it is a concern that the molten metal tends to drip from both sides of the plate. Due to the thinness of the plate, it is difficult for the molten metal to remain on the upper surface of the end portion. In order to prevent the molten metal from dripping, it is necessary that the deposition width not be too large compared to the plate thickness, i.e., that the amount of material supplied is not excessive. In addition, the amount of molten metal is considered to become large because the heat capacity is small, and the temperature at the fabrication zone rises easily. To prevent the amount of molten metal from becoming too large, it is necessary to keep the heat input sufficiently low. It is therefore necessary to estimate the deposition width from the deposition conditions beforehand 
and perform the additive fabrication under the appropriate deposition conditions, including a deposition width suitable for the plate thickness. It is necessary to grasp the causal relationship between the deposition width and the deposition conditions such as the material supply, heat input, and interlayer temperature. In WAAM, the lower the interlayer temperature, the faster the cooling speed of the subsequent layer. When the cooling speed is fast, it increases the degree of supercooling when the molten pool cools down, and the solidifying temperature falls, the surface tension at solidification heightens, and the contact angle becomes larger. With a larger contact angle, the length of the area of contact with solids at the liquid phase becomes smaller and the deposition width becomes smaller (Kamioka et al., 2012). By monitoring the interlayer temperature and controlling the interval time so that the interlayer temperature remains constant, the temperature state of each layer deposited is considered to become constant, and a uniform deposition width can be obtained.

First, we fabricated wall structures on the flat substrates because no dripping of molten metal occurred while keeping the interlayer temperature constant, and investigated the relationship between the interlayer temperature and the deposition width. These structures were different in shape and thermal capacity from the structure on the thin end of the plate that we intended to fabricate. Therefore, the conditions needed to be adjusted when the additive fabrication onto the thin end of the plate was performed. Table 1 lists the experimental conditions. In the experiment, SUS304 was used instead of Inconel 718 as the substrate because the mechanical properties of the boundary between the substrate and additive fabricated part were not evaluated in this study, and the influence of the shape and thermal characteristics would be the dominant factor in the experiment. In preliminary experiments, almost similar deposition shapes were obtained in the cases of fabrication with Inconel 718 SUS304 as a substrate. Then SUS304 was used to conduct more experiments considering the economics of the experiment. We defined the fabrication cross-section $S$ as the material supply per minute divided by the migration length per minute, so as to show the relationship between the deposition conditions and the deposition width $w$. The fabrication cross-section $S$ is the cross-section per layer of the fabrication. The fabrication cross-section $S\left[\mathrm{~mm}^{2}\right]$ is expressed as

$$
S=10^{3} F_{W} \cdot \pi(D / 2)^{2} / F
$$

where $F_{W}[\mathrm{~m} / \mathrm{min}]$ is the wire feed speed, $D[\mathrm{~mm}]$ is the wire diameter, and $F[\mathrm{~mm} / \mathrm{min}]$ is the torch feed speed. In addition, the heat input $H[\mathrm{~J} / \mathrm{mm}]$, which is applied to the weld zone from the power source during fabrication, is expressed as

$$
H=60 I V I F
$$

where $I[\mathrm{~A}]$ is the welding current, and $V[\mathrm{~V}]$ is the arc voltage. Generally, in semiautomatic MIG/MAG welding, the welding machine automatically sets the wire feed speed $F_{W}$ according to the welding current $I$ value. $F_{W}$ is in direct proportion to the welding current $I$. Therefore, as the fabrication cross-section $S$ becomes larger, so does the heat input $H$. In this method, however, the appropriate heat quantity is automatically set according to the material supply. The wire feed speed $F_{W}$ and the torch feed speed $F$ in Table 1 were determined by combinations with certain kinds of fabrication cross-section $S$ values. The wall structures were fabricated by depositing 10 layers of welding beads, $100 \mathrm{~mm}$ in the horizontal direction, onto the substrate. The deposition widths $w$ of the fabricated wall structures were measured with a vernier caliper and taken the average. The measurement positions of the deposition width $w$ were $-40 \mathrm{~mm},-20 \mathrm{~mm}, 0$ $\mathrm{mm},+20 \mathrm{~mm}$ and $+40 \mathrm{~mm}$, where the center of the fabricated wall structure of $100 \mathrm{~mm}$ in length was set to 0 . Due to the characteristics of the welding bead, the deposition height of the welding start position was different from that of the finish position. Then, the difference in the deposition height between the welding start position and the finish position was offset by alternately changing the torch feed direction for each layer.

Figure 5 shows the relationship between the measured deposition width $w$ and the fabrication cross-section $S$. As the fabrication cross-section $S$ increased, the deposition width $w$ became larger. By comparing the influence of the interlayer temperature under the condition in which the fabrication cross-section $S$ is equal, the deposition width $w$ tends to become larger with a higher interlayer temperature. As the fabrication cross-section $S$ becomes larger, the influence of the interlayer temperature on the deposition width $w$ increases. In addition, the relationship between the deposition width $w$ and the fabrication cross-section $S$ at each interlayer temperature can be approximated by a power relationship. That power approximation is expressed as

$$
w=a S^{b}
$$

where $a$ and $b$ are coefficients that change according to the interlayer temperature. From Eqs. (1) and (3), the deposition 
width $w$ is expressed as

$$
w=a\left(10^{3} F_{W} \cdot \pi(D / 2)^{2} / F\right)^{b}
$$

It was found that the deposition width $w$ is determined uniquely by the combination of the wire feed speed $F_{W}$ and the torch feed speed $F$, which are the deposition conditions set at the time of fabrication.

Table 1 Experimental conditions of wall structure

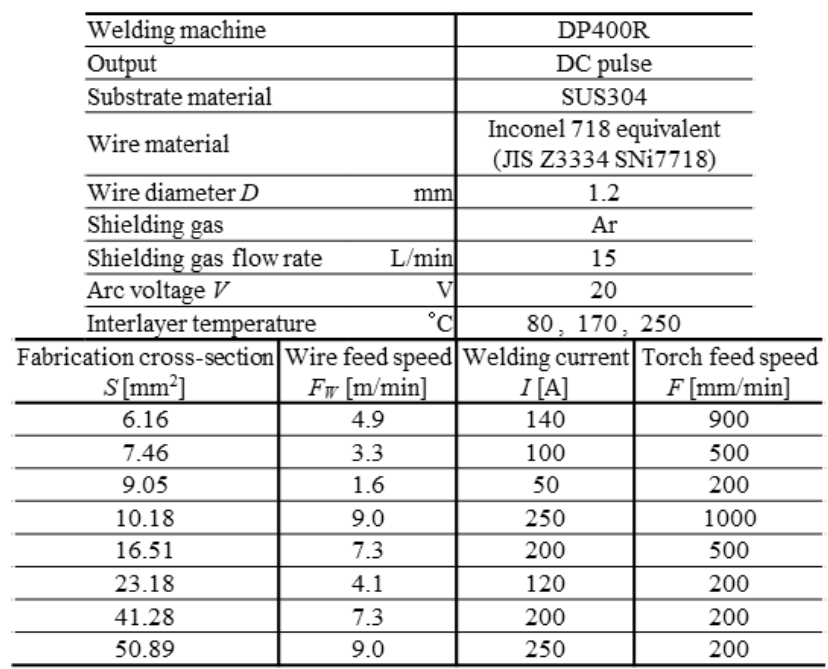

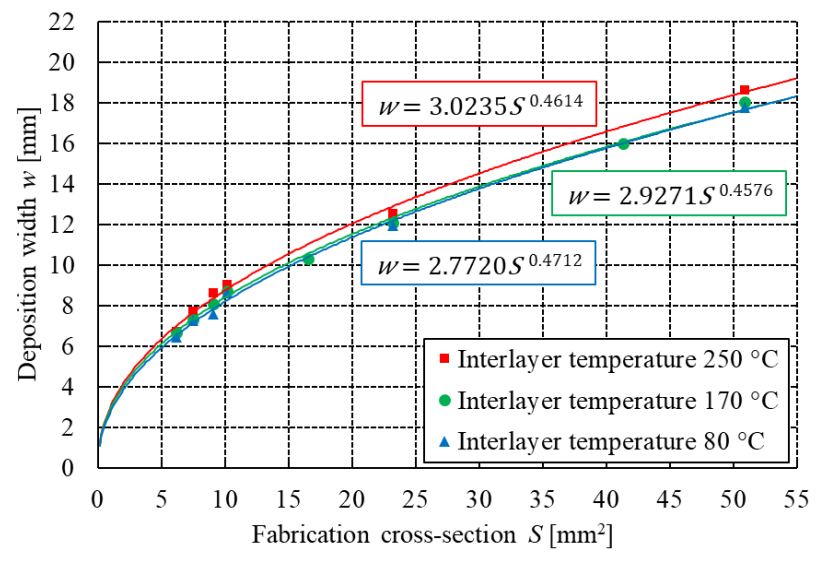

Fig. 5 Relationship between deposition width and fabrication cross-section

\section{Additive fabrication onto the thin end of the plate}

In this chapter, we propose a method to determine the deposition conditions of wire feed speed $F_{W}$ and torch feed speed $F$ for obtaining the target deposition width $w$ using the relationship between the deposition conditions and the deposition width $w$ obtained by the interlayer temperature monitoring shown in the previous section. From Eq. (3), the fabrication cross-section $S$ is expressed as

$$
S=(w / a)^{1 / b}
$$

Using Eq. (5), the fabrication cross-section $S$ is obtained by substituting the target deposition width $w$. From Eq. (1), the wire feed speed $F_{W}$ is expressed as

$$
F_{W}=S F /\left(10^{3} \cdot \pi(D / 2)^{2}\right)
$$

Using Eq. (6), the wire feed speed $F_{W}$ is obtained by substituting the fabrication cross-section $S$ obtained from Eq. (5) and the arbitrary torch feed speed $F$. In that case, it is generally better to set the torch feed speed $F$ higher, so as to increase the fabrication efficiency.

In this experiment, thin plates (plate thickness $t_{p}: 1,3$, and $5 \times 50 \times 30 \mathrm{~mm}$ ) of Inconel 718 were prepared, and additive fabrications of 10 layers were performed. The interlayer temperature was kept constant at $170{ }^{\circ} \mathrm{C}$. That is, using the approximate curve of the interlayer temperature of $170{ }^{\circ} \mathrm{C}$, the equation of the relationship between the deposition width $w$ and the fabrication cross-section $S$ is expressed as

$$
w=2.9271 S^{0.4576}
$$

For plate thicknesses $t_{p}$ of 1,3 , and $5 \mathrm{~mm}$, the target deposition width $w$ of the fabricated wall structure was set to $3.84,4.80$, and $6.41 \mathrm{~mm}$, respectively. Considering that the same width as the plate thickness $t_{p}$ is obtained after the finish cut, each target deposition width $w$ was set to about 1 to $3 \mathrm{~mm}$ larger than each plate thickness $t_{p}$. The fabrication cross-section $S$ and the wire feed speed $F_{W}$ were calculated using Eqs. (5) and (6), and the torch feed speed $F$ was 1000 $\mathrm{mm} / \mathrm{min}$, which is the upper limit of the machine, so as to increase the fabrication efficiency. Table 2 lists the experimental conditions defined for each plate thickness $t_{p}$. In cases where the wire feed speed $F_{W}$ is smaller than 1.6 $\mathrm{m} / \mathrm{min}$ (i.e., the welding current $I$ is lower than $50 \mathrm{~A}$ ), the arc may not be generated and fabrication cannot be performed. In such cases, the lower limit of the wire feed speed $F_{W}$ is set to $1.6 \mathrm{~m} / \mathrm{min}$. When the torch feed speed $F$ is $1000 \mathrm{~mm} / \mathrm{min}$, which is the upper limit of the machine, and the wire feed speed $F_{W}$ is $1.6 \mathrm{~m} / \mathrm{min}$, which is the lower 
limit, using Eqs. (1) and (7), the minimum deposition width in the machine is $3.84 \mathrm{~mm}$, which is about $3 \mathrm{~mm}$ larger than the plate thickness of $1 \mathrm{~mm}$. The wall structures were fabricated by depositing 10 layers of welding beads $50 \mathrm{~mm}$ in length in the horizontal direction onto the thin end of the plate. The deposition widths $w$ of the fabricated wall structures were then measured.

Figure 6 shows the wall structures fabricated of 10 layers on the thin end of the plate for each plate thickness and the thermal images of the fabricated wall structures at the tenth layer. The thermal images in Fig.6 were the temperature measured at the timing when the interlayer temperature of the top bead fell to $170{ }^{\circ} \mathrm{C}$ after depositing the 10 th layers. After the fabricating, the color on the surface around the boundary between the substrate and the deposited layers turned dark by the effect of the fume and oxidation. Then the emissivity at this part became higher than the part just after the deposition, so the temperature seemed higher than the actual temperature.

It was confirmed that additive fabrication could be performed onto the thin end of the plate for all plate thicknesses without molten metal dripping. Stable wall structures were fabricated onto the thin end of the plate at plate thicknesses of 3 and $5 \mathrm{~mm}$. On the other hand, the wall structure fabricated on the thin end of the 1-mm-thick plate was unstable. This was because the welding bead shape becomes unstable when the plate thickness is small and the heat capacity is small, and the amount of molten metal becomes large under deposition conditions of the minimum deposition width of $3.84 \mathrm{~mm}$ in the machine, that is, $50 \mathrm{~A}$, which is the lower limit of the welding current I at which the arc can be generated. Laminating the unstable welding beads causes the final fabricated wall shape to also become unstable. It is considered necessary to further increase the torch feed speed $F$ so as to reduce the amount of molten metal deposited onto the thin end of the plate in order to stabilize the fabricated wall structure.

Figure 7 shows the target value and measured value of the deposition width $w$ of the fabricated wall structure of 10 layers on the thin end of the plate for each plate thickness. The deposition widths $w$ of the fabricated wall structures were measured with a vernier caliper and taken the average. The measurement positions of the deposition width $w$ were $-15 \mathrm{~mm},-7.5 \mathrm{~mm}, 0 \mathrm{~mm},+7.5 \mathrm{~mm}$ and $+15 \mathrm{~mm}$, where the center of the fabricated wall structure of $50 \mathrm{~mm}$ was set to 0 . The measured value of the deposition width was $0.16 \mathrm{~mm}$ larger than the target value of the deposition width at the plate thickness of $1 \mathrm{~mm}$. However, it was possible to roughly fabricate a wall structure with the target deposition width on the thin end of the plate at all plate thicknesses. It was shown that it is effective to determine the deposition conditions at the time the wall structure is fabricated on the thin end of the plate by using the relationship between the deposition conditions and the deposition width $w$ obtained by interlayer temperature monitoring.

Next, additive fabrications were performed with the target deposition widths of 5, 6, 7, and $8 \mathrm{~mm}$, assuming that cutting was applied after the additive fabrication was done and after finishing to a surface precision that was the same as that before the repair. More appropriate deposition widths were investigated for a plate thickness of $5 \mathrm{~mm}$. Table 3 lists the experimental conditions. In this experiment, thin plates (plate thickness $t_{p}: 5 \times 50 \times 30 \mathrm{~mm}$ ) of SUS304 were prepared. Assuming that the same width as the plate thickness $t_{p}$ was to be obtained after cutting, the target deposition $w$ was set to be about +0 to +3 mm larger than the plate thickness $t_{p}$. For each target deposition width $w$, the fabrication cross-section $S$ and the deposition width $w$ were calculated from Eq. (7). The wall structure was fabricated by depositing 10 layers of welding beads onto the thin end of the plate. The depositing width $w$ of the fabricated wall structure was then measured.

Figure 8 shows the fabricated wall structures of 10 layers on the thin end of the plate under each condition. It was confirmed that additive fabrication could be performed onto the thin end of the plate under all conditions without molten metal dripping.

Figure 9 shows the target value and measured value of the deposition width $w$ of the fabricated wall structure of 10 layers on the thin end of the plate under each condition. Under conditions 1 and 2, the measured value was clearly smaller than the target value. This was because the welding current $I$ was set to be low because the wire feed speed $F_{W}$ was low, the heat input was small for the heat capacity of the plate thickness, and a thin bead was fabricated. In order to increase the wire feed speed $F_{W}$ with the same target deposition width, the head feed $F$ speed also needed to be increased. However, in this machine, the upper limit of the head feed speed $F$ is $1000 \mathrm{~mm} / \mathrm{min}$, and the wire feed speed $F_{W}$ could not be further increased because the head feed speed $F$ could not be further increased. Under conditions 3 and 4 , it was possible to roughly fabricate the wall structure with the target deposition width on the thin end of the plate. From the above results, it was found that it is necessary to perform fabrication with a target deposition width with +2 $\mathrm{mm}$ or more for the plate thickness in order to obtain sufficient heat input for the heat capacity of the plate when performing additive fabrication onto the thin end of the plate with the target deposition width. 
Tsurumaki, Tsukamoto, Chibahara and Sasahara,

Journal of Advanced Mechanical Design, Systems, and Manufacturing, Vol.13, No.2 (2019)

Table 2 Experimental conditions of wall structure on the thin ends of plates of different plate thicknesses

\begin{tabular}{c|c|c|c|}
\hline $\begin{array}{c}\text { Plate thickness } \\
t_{p}[\mathrm{~mm}]\end{array}$ & $\begin{array}{c}\text { Fabrication cross-section } \\
S\left[\mathrm{~mm}^{2}\right]\end{array}$ & $\begin{array}{c}\text { Wire feed speed } \\
F_{W}[\mathrm{~m} / \mathrm{min}]\end{array}$ & $\begin{array}{c}\text { Welding current } \\
I[\mathrm{~A}]\end{array}$ \\
\hline 1 & 1.81 & 1.6 & 50 \\
\hline 3 & 2.94 & 2.6 & 80 \\
\hline 5 & 5.54 & 4.9 & 140 \\
\hline
\end{tabular}

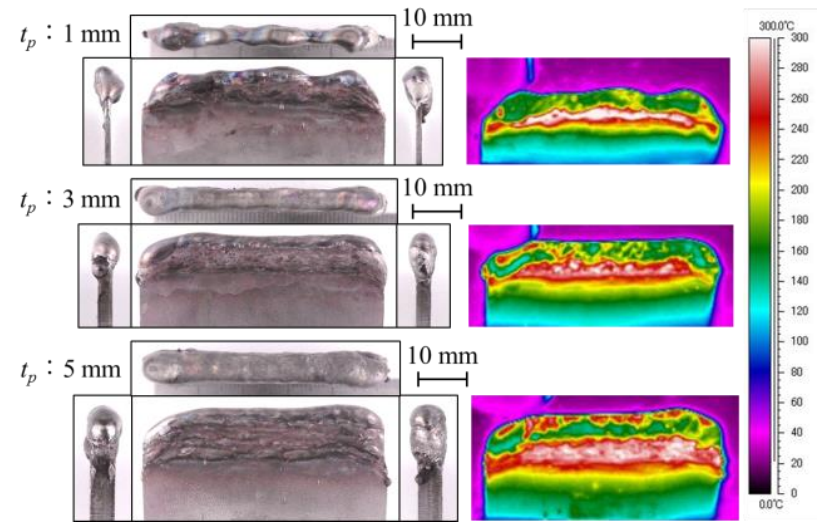

Fig. 6 Wall structure on the thin end of the plate and thermal images at an interlayer temperature of $170{ }^{\circ} \mathrm{C}$

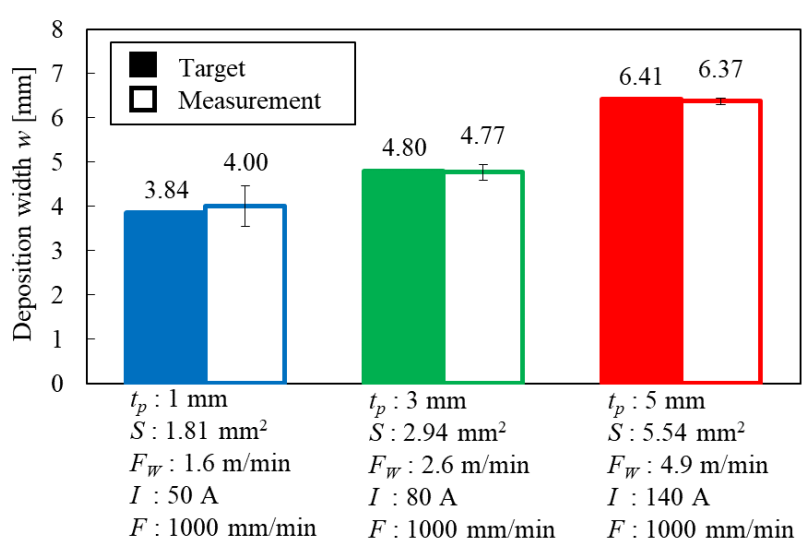

Plate thickness $t_{p}[\mathrm{~mm}]$, Fabrication cross-section $S\left[\mathrm{~mm}^{2}\right]$, Wire feed speed $F_{W}[\mathrm{~m} / \mathrm{min}]$, Welding current $I[\mathrm{~A}]$, Torch feed speed $F[\mathrm{~mm} / \mathrm{min}]$

Fig. 7 Deposition width of wall structure on the thin ends of plates of different plate thicknesses

Table 3 Experimental conditions of wall structure on the thin ends of plates of the same plate thickness

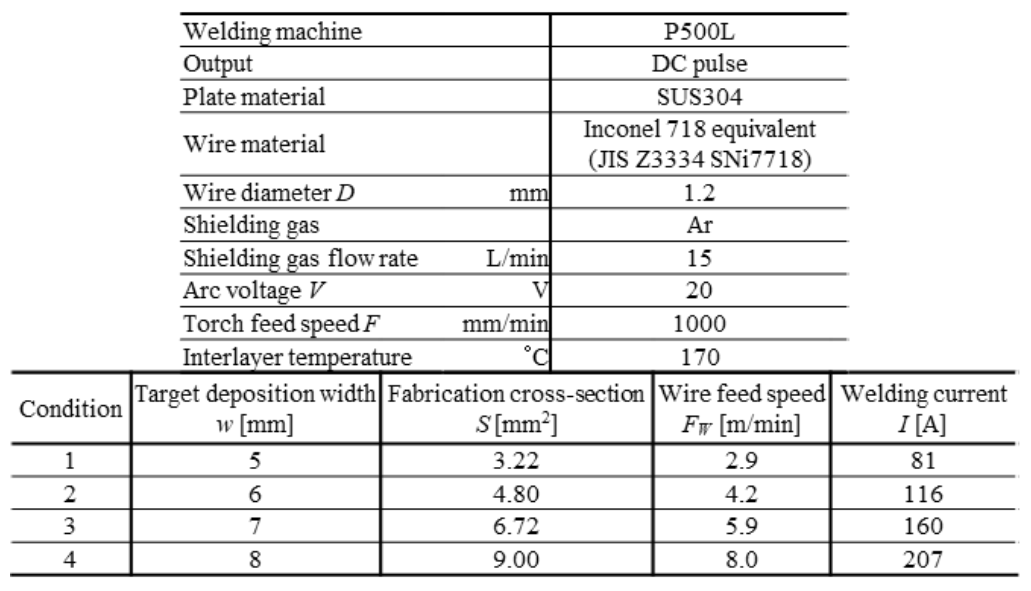

Condition : 1

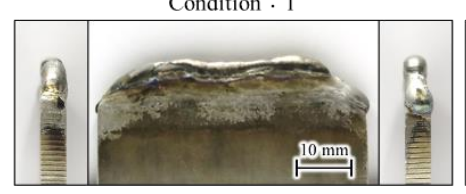

Condition : 3

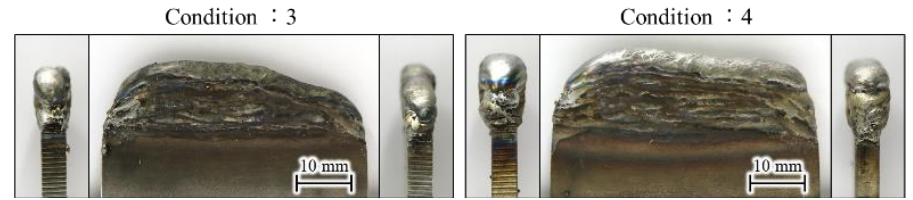

Fig. 8 Wall structure on the thin ends of plates of the same plate thickness
Condition $: 2$

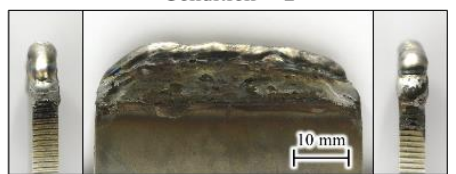

Condition : 4

$10 \mathrm{~mm}$

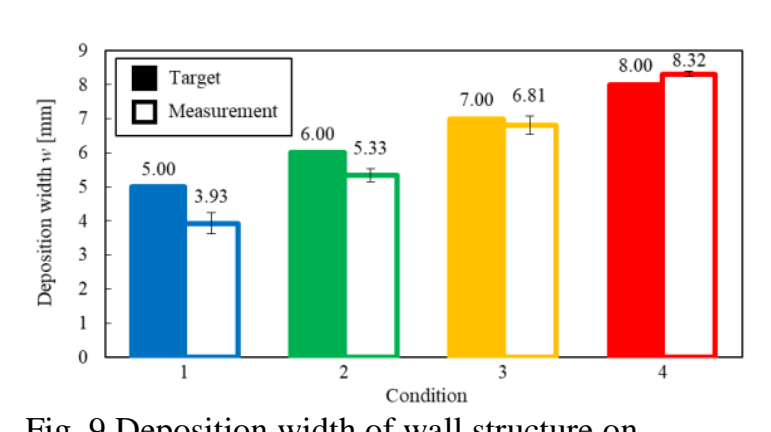

Fig. 9 Deposition width of wall structure on the thin ends of plates of the same plate thickness 


\section{3D scan and cutting of additive fabrication onto the thin end of the plate}

In this chapter, three-dimensional measurements were performed on the fabricated wall structure of 10 layers on the thin end of the plate in the previous section before cutting. For three-dimensional measurement, a 3D-scanner-type three-dimensional measurement machine (KEYENCE VL-300) was used.

Using the 3D comparative measurement function of the 3D-scanner-type three-dimensional measurement machine, a height comparison was made based on the finished surface after the side cutting was applied to the surface of the thin plate and the fabricated wall structure. Figure 10 shows the result of a 3D comparison of the fabricated wall structures under four conditions. The colors of the surface of the fabricated wall structure indicate the height difference between the finished surface and the surface of the fabricated wall structure. The colored parts of the area where the surface color of the fabricated wall structure shows a negative value indicate uncut parts where the surface of the fabricated wall structure is inside the surface to be cut. This means that even after the finish cutting, a desiable surface accuracy can not be obtained. In conditions 1 and 2, the colors of the negative area, including the green color, can be widely confirmed on the surface of the fabricated wall structure, and it is assumed that they are left as uncut parts. In condition 3 , the colors of the negative area were confirmed in just a few places. On the other hand, in condition 4 , there was almost no negative area, and it was possible to obtain the assumed surface accuracy by cutting the surface of the fabricated wall structure.

Next, side cutting of the fabricated wall structure on the thin end of the plate was performed to confirm whether additive fabrication can be performed without defects. For the side cutting, a 4-flute radius end mill with a diameter of $10 \mathrm{~mm}$ was used as a tool.

Figure 11 shows the fabricated wall structures of 10 layers under four conditions under which side cutting was applied to the surface of the thin plate. Under all of the conditions, there was no deflection after cutting and no deformation due to residual stress. Under conditions 1, 2, and 3, there were uncut parts, where the surface color remained black on the fabricated wall structure because the surface of the fabricated wall structure was inside the plate surface. Under condition 4 , additive fabrication could be performed with no defects by setting conditions at the target deposition width $w$ of $8 \mathrm{~mm}$ or more for a plate thickness of $5 \mathrm{~mm}$ under which the molten metal did not drip.
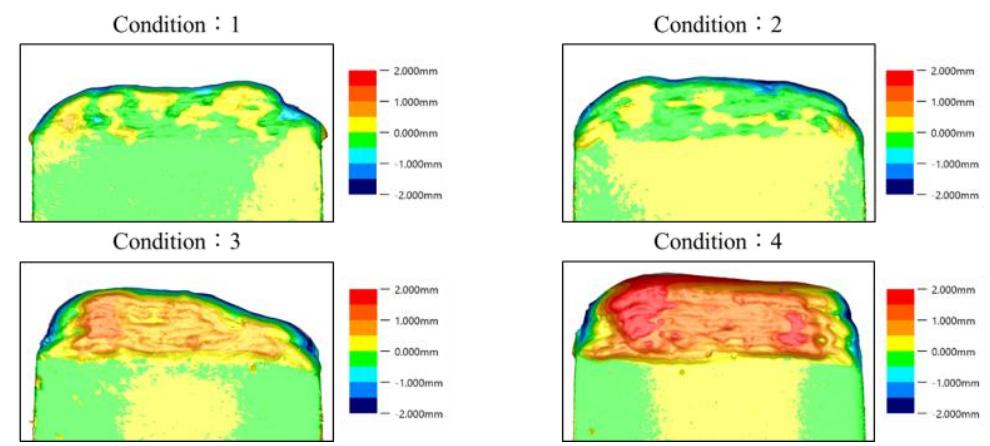

Fig. $103 \mathrm{D}$ comparison of wall structures on the thin end of the plate

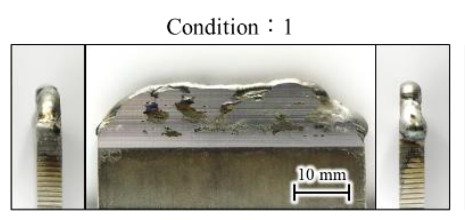

Condition : 3

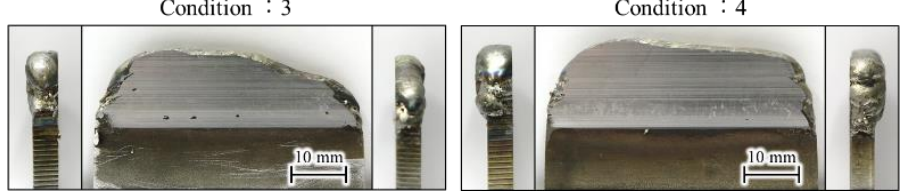

Condition : 2

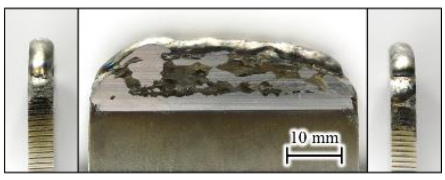

Condition : 4

Fig. 11 Wall structure with side cutting applied on the thin end of the plate 


\section{Conclusions}

We performed additive fabrication onto the thin end of a plate and finish cutting based on the relationship between deposition conditions and deposition width by monitoring the interlayer temperature using Inconel 718. Our findings can be summarized as follows.

(1) When the interlayer temperature is kept constant with temperature monitoring, the relationship between the deposition width and the fabrication cross-section can be approximated by a power relationship. As a result, we showed that deposition width is uniquely determined from the combination of wire feed speed and torch feed speed, which are the deposition conditions set at the time of fabrication.

(2) We demonstrated that additive fabrication of a wall structure with the aimed deposition width can be deposited onto the thin end of a plate of any thickness from $1.0 \mathrm{~mm}$ to $5.0 \mathrm{~mm}$ using the relationship between the deposition conditions and the deposition width, obtained by monitoring the interlayer temperature.

(3) It is desirable to perform additive fabrication onto the thin end of a plate under conditions in which a target lamination width $w$ of $8 \mathrm{~mm}$ or more does not cause dripping of molten metal with a plate thickness of $5 \mathrm{~mm}$. It is possible to obtain the desired surface accuracy by cutting the surface of the fabricated wall structure.

\section{References}

Abe, T., and Sasahara, H., Dissimilar metal deposition with a stainless steel and nickel-based alloy using wire and arc-based additive manufacturing, Precision Engineering, Vol.45, (2016), pp. 387-395.

Bi, G. and Gasser, A., Restoration of nickel-base turbine blade knife-edges with controlled laser aided additive manufacturing, Physics Procedia, Vol.12, (2011), pp.402-409.

Carter, T. J., Common failures in gas turbine blades, Engineering Failure Analysis, Vol.12, (2005), pp. $237-247$.

del Campo, L., Pérez-Sáez, R.B., González-Fernández, L., Esquisabel, X., Fernández, I., González-Martín, P., Tello, M.J., Emissivity measurements on aeronautical alloys, Journal of Alloys and Compounds, Vol. 489, Issue 2, (2010), pp.482-487.

Funderburk, R. S., The importance of interpass temperature, Welding Innovation, Vol.XV, No.1, (1998).

Kamioka, T., Abe, T., Ishikawa, S., and Sasahara, H., Influence of the cooling method of the molten pool on the laminating characteristics in direct metal lamination by using arc discharge, Transactions of the Japan Society of Mechanical Engineers, Series C, Vol.78, No.785, (2012), pp.282-291 (in Japanese).

Liu, W. D., Ao, S. S., Li, Y., Zhao, C. F., Luo, Z., Li, Q. and Luo, T., Elimination of the over cut from a repaired turbine blade tip post-machined by electrochemical machining, Journal of Materials Processing Technology, Vol. 231, (2016), pp.27-37.

Pallos, K. J., Gas Turbine Repair Technology, GE Energy Services Technology, Atlanta, GA, GER-3957B, (2001), pp. 7-10.

Petro, J. S., Effect of interpass temperature on the structure and properties of multipass weldments in high performance nickel alloys, CSU Theses and Dissertations, Colorado State University, (2011).

Rani, S., Agrawal, A. K. and Rastogi, V., Failure analysis of a first stage IN738 gas turbine blade tip cracking in a thermal power plant, Case Studies in Engineering Failure Analysis, Vol. 8, (2017), pp. 1-10.

Thomas, A., El-Wahabi, M., Cabrera, J. M. and Prado, J. M., High temperature deformation of Inconel 718, Journal of Materials Processing Technology, Vol. 177, Issues 1-3, (2006), pp.469-472. 